\title{
Gradhiva
}

GRADHI

Revue d'anthropologie et d'histoire des arts

26 | 2017

En croire ses sens

\section{Introduction. Sensorialités religieuses : sens, matérialités et expériences}

Anouk Cohen, Katerina Kerestetzi et Damien Mottier

\section{(2) OpenEdition}

\section{Journals}

Édition électronique

URL : http://journals.openedition.org/gradhiva/3418

DOI : 10.4000/gradhiva.3418

ISSN : 1760-849X

Éditeur

Musée du quai Branly Jacques Chirac

Édition imprimée

Date de publication : 6 décembre 2017

Pagination : 4-21

ISBN : 978-2-35744-096-8

ISSN : 0764-8928

\section{Référence électronique}

Anouk Cohen, Katerina Kerestetzi et Damien Mottier, «Introduction. Sensorialités religieuses : sens, matérialités et expériences », Gradhiva [En ligne], 26 | 2017, mis en ligne le 06 décembre 2017, consulté le 23 septembre 2020. URL : http://journals.openedition.org/gradhiva/3418; DOI : https://doi.org/ 10.4000 /gradhiva.3418 


\section{Introduction}

\section{Sensorialités religieuses: sens, matérialités et expériences}

par Anouk Cohen, Katerina Kerestetzi et Damien Mottier

Thomas le sceptique, qui était absent le soir de la résurrection quand Jésus apparut aux disciples, douta de leur témoignage: «Si je ne vois dans ses mains la marque des clous, et si je ne mets mon doigt dans la marque des clous, et si je ne mets ma main dans son côté, je ne croirai point. " Huit jours plus tard, Jésus leur apparut de nouveau dans une pièce fermée. Cette fois, Thomas était parmi eux: «Avance ici ton doigt, et regarde mes mains; avance aussi ta main, et mets-la dans mon côté; et ne sois pas incrédule, mais crois. " Dans un célèbre tableau de 1603 - L'Incrédulité de saint Thomas - consacré à ce passage de l'évangile de Jean (Jean 20. 19-29), Le Caravage représente Thomas plongeant son doigt dans la chair de ce Dieu-fait-homme pour éprouver les stigmates et orienter les regards vers une théologie de l'Incarnation qui est au fondement du christianisme.

La compréhension sensible de ce rapport chrétien au monde se joue par un subtil recouvrement du visible par le tactile. Toucher est une expérience de la limite, une manière de mettre en relation le visible et l'invisible, le sensible et l'intangible (Nancy 1993). Mais le toucher n'est jamais univoque. Car ce qui touche est nécessairement touché-touchant. Bien qu'éloignés du monde chrétien, les initiés du palo monte afro-cubain expérimentent le divin en des termes qui ouvrent la réflexion à une étonnante comparaison. Les pratiquants de ce culte des morts ne sont pas tenus d'y croire. Au contraire, il est courant de les entendre dire: « Nous sommes comme saint Thomas! Voir pour croire! Et je ne crois que ce que je vois! » Une grande partie de leur activité rituelle consiste à rendre sensibles les morts et les dieux en leur attribuant une forme de corporéité, une odeur, une voix. L'efficacité de ces entités invisibles repose sur leur capacité à être vues, senties et entendues, «à donner des preuves", déclarent les initiés. Ainsi, le palo monte fournit un autre exemple de la façon dont le croire s'articule avec le percevoir et le sentir, sinon en découle.

Ce numéro propose d'explorer la dimension polysensorielle de l'expérience et de la subjectivité religieuses. Loin de seulement examiner comment la sensorialité s'enchevêtre avec l'activité religieuse, il s'attache à montrer que celle-ci est une expérience sensible. À travers une approche pragmatique, chacune des contributions questionne les modalités suivant lesquelles des activités rituelles sont modelées et (re)produites par une combinaison des sens engagés dans la matière. Quels perceptions, sensations, émotions et mouvements naissent-ils de la rencontre avec les objets, les substances et l'environnement physique d'une pratique religieuse? Comment le corps sensible et la matérialité d'une pratique religieuse s'activent-ils mutuellement au cours de l'activité rituelle? Dans quelle mesure leurs imbrication et articulation structurent-elles diversement la formation et la transmission des savoirs? 


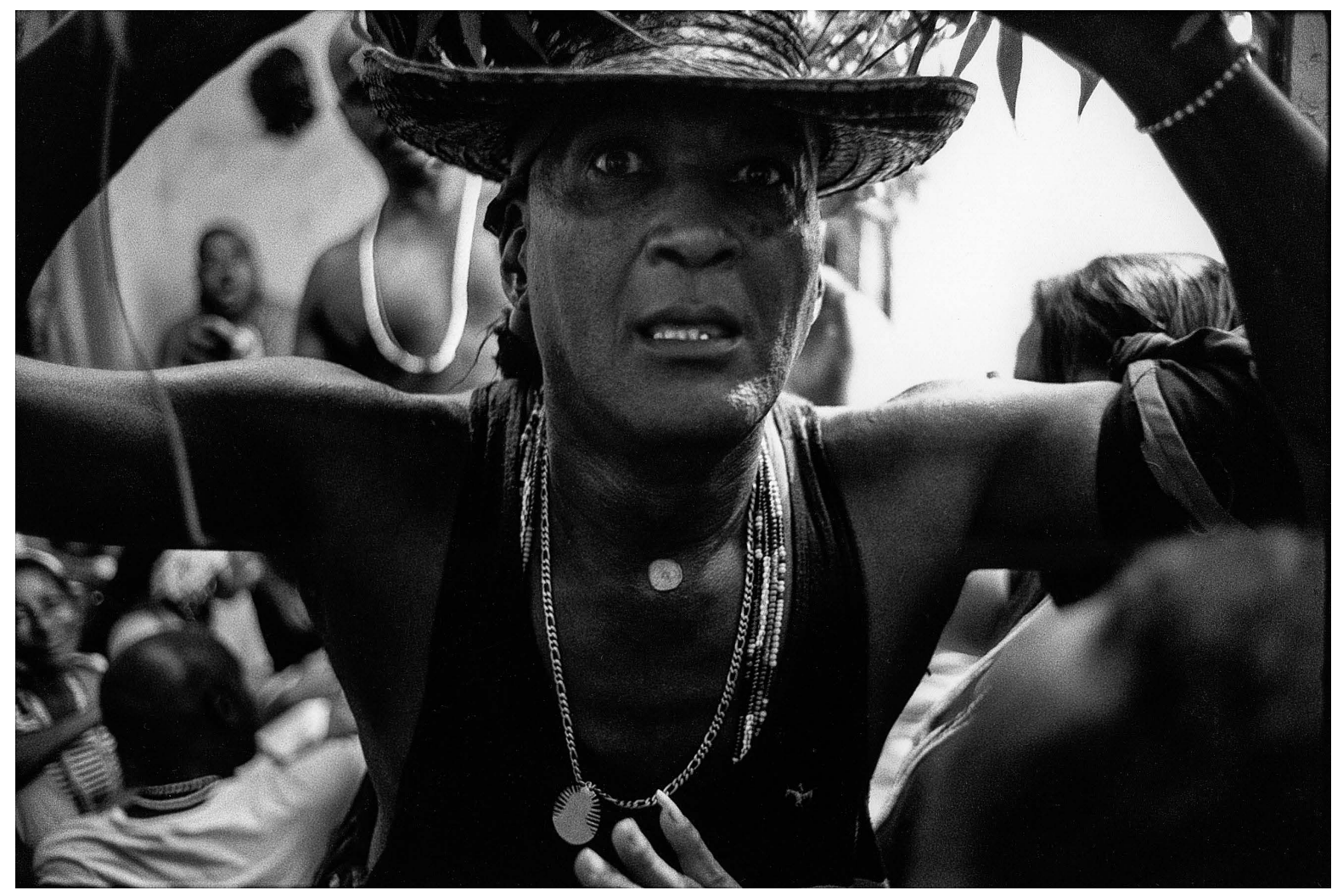

fig. 1

Claire Garoutte

et Anneke Wambaugh,

Santiago Castaneda

Vera accueille l'esprit

de Pa Francisco, 2000

(c) Garoutte/Wambaugh,

Crossing the Water:

A Photographic Path to the

Afro-Cuban Spirit World.

Université du Michigan,

Duke University Press,

2007. 
À partir d'ethnographies détaillées, les contributeurs examinent comment les pratiquants vivent leur religiosité à travers des comportements ordinaires - marcher, toucher, réciter, lire, entendre, voir - en composant avec des contraintes relationnelles, économiques, politiques et spatiales ancrées dans des univers religieux distincts: islam, pentecôtisme, chamanisme, animisme, catholicisme. L'attention est portée aussi bien aux cinq sens qu'à l'électroperception, ou encore à la synesthésie: aucun des sens - non seulement la vue, l'odorat, l'ouïe, le goût, le toucher mais aussi le sens kinesthésique, le sens viscéral - ne peut être isolé. Ils se recouvrent nécessairement les uns les autres: un son peut évoquer une image, le goût une odeur, et inversement. La question de la combinaison des sens et de leur ordonnancement a constitué un point de départ stimulant pour notre analyse. Car de cet agencement méthodique et de ces relations dépend une meilleure compréhension des configurations du sensible qu'il appartient à l'ethnographe de décrire. Cela conduit à opérer un basculement du croire au faire sinon au (res)sentir. À cette fin, sont principalement étudiés dans ce numéro l'action et l'interaction des humains et des non-humains, les espaces liturgiques et l'éducation corporelle des pratiquants, de manière à appréhender comment les sens agissent, se manifestent et sont discrètement fabriqués, notamment rituellement et politiquement.

Tandis que les sensations sont généralement envisagées comme des phénomènes intimes et privés, nous souhaitons ici les resituer dans un régime collectif de sens. Au croisement des approches phénoménologiques, cognitivistes et culturalistes, notre ambition est d'approfondir la réflexion sur la façon dont l'idiosyncrasie religieuse se mue en tradition partagée. D'où l'importance d'enrichir notre compréhension du rôle des corps et des sens dans la production de l'expérience religieuse en analysant comment ils interagissent avec la matière, source de sensations, d'émotions et d'imagination rituelle.

\section{Sentir: pratique, engagement et synesthésie}

La question des sens comme objet d'étude anthropologique s'est progressivement imposée dans les années 1990 (Stoller 1989; Howes 1991; Classen 1993) et connaît une certaine actualité en France (Candau 2000; Colon [dir.] 2013; Laplantine 2005; Whatelet 2009; Candau et Wathelet 2013) ${ }^{1}$. Le sensory turn a émergé du constat simple et édifiant qu'on ne peut appréhender la diversité culturelle sans considérer les manières dont les différentes sociétés établissent des régimes sensoriels propres (Howes 2003). Ainsi, l'enjeu d'une analyse anthropologique des sens a été bâti, en relation avec l'histoire des sensibilités (Corbin 1982 et 1994 notamment; voir également dans ce dossier l'entretien mené par les anthropologues Emma-Aubin Boltanski et Giordana Charuty avec I'historien médiéviste Jean-Claude Schmitt), sur un travail de comparaison s'attachant à rendre intelligible la variabilité des régimes sensoriels et leur hiérarchisation, soit à saisir la façon dont tous les sens sont culturellement agencés et discriminés. Plus précisément, il s'agit de révéler «l'influence que de telles variabilités ont sur les formes d'organisation sociale, sur les conceptions du soi et du cosmos, sur la régulation des émotions et sur d'autres domaines d'expression culturelle " (Howes 1991: 3, trad. des auteurs). Cette anthropologie culturaliste des sens s'emploie à dégager des «profils sensoriels » (sensory profiles) et des «ordres sensoriels » (sensory
1. Pour une synthèse récente, voir Gélard 2016a; $2016 b$. 
2. Voir notamment Keane 2007, Morgan 2010 et Bynum 2011. Et pour une synthèse récente: Cohen et Mottier 2016 orders) [ibid.] socialement construits et analysés du point de vue discursif plutôt qu'en fonction de la dimension processuelle et performative des sens à une échelle individuelle et collective.

Or, comme le remarque Tim Ingold (cité par Pink 2009), le corps ne peut pas être considéré comme seul révélateur des représentations liées aux sens et à leur valorisation culturelle. Le corps, défend-il, est un corps engagé. Ainsi la phénoménologie et les sciences cognitives, comme les approches qui s'en inspirent dans les domaines de la psychologie (Gibson 1977), de la géographie (Rodaway 1994; Tuan 1975) ou de la danse (Godard 1995), se sont rapidement constituées en un arrière-plan indispensable au renouvellement de la réflexion anthropologique sur les sens. S'il ressort de la confrontation de ces différentes approches un contraste entre les démarches focalisées sur l'individu et d'autres centrées sur le groupe, celles-ci ne doivent toutefois pas être opposées. Des chercheurs d'inspiration cognitiviste ou phénoménologique se sont en effet penchés sur les questions sociales - Levinson (2003), par exemple, insiste sur le conditionnement culturel du sens de l'orientation - et des chercheurs culturalistes se sont intéressés aux mécanismes naturels de la construction individuelle des sens (voir, par exemple, Hall 1974).

Dans le prolongement de ces débats, ce dossier conjugue l'analyse des rapports à soi et au collectif. II entend à la fois cerner les modes d'engagement corporel dans l'activité cultuelle et saisir leur impact sur sa configuration. S'appuyant sur les récents développements d'une approche matérielle des pratiques religieuses ${ }^{2}$, il s'agit en particulier de comprendre comment celles-ci sont façonnées et modélisées par les sens, eux-mêmes stimulés par des objets, des substances, des sons et des formes. Ces expériences peuvent ainsi être définies comme des configurations du sensible, soit des activités servies par la mobilisation soigneusement maîtrisée des différents registres sensoriels, à partir de matérialités agissantes. Chacune à leur manière, les contributions ici réunies portent sur la façon dont les individus éprouvent leur engagement religieux et dont la codification de l'action rituelle crée les conditions d'une sensibilité partagée. Le corps des pratiquants, point nodal de l'interaction avec l'environnement physique, social et relationnel du culte, est donc placé au centre de l'analyse. Trois principaux axes thématiques se dégagent de ce dossier: mémorisation, spatialisation et normalisation.

\section{Mémoriser: corps et productions}

Considérant que la transmission des pratiques religieuses est prioritairement liée à leur mémorisation, Harvey Whitehouse a distingué deux modes de religiosité - doctrinal (caractérisé par une liturgie stabilisée et routinière) et imagistique (plus performatif, avec des rituels marquant et sensoriellement forts) - qui correspondent à deux modes de mémorisation - sémantique et épisodique (Whitehouse 2000; 2004). Plusieurs chercheurs s'accordent cependant à dire que ces deux modes n'épuisent pas les rapports différenciés à la religiosité (Laidlaw et Whitehouse [dir.] 2004; Berliner et Sarró [dir.] 2007), ce qui ne diminue pas la pertinence de la théorie de Whitehouse, qui vient compléter les approches sémiotiques et linguistiques en insistant sur le rôle du corps sensible mémorisant dans les pratiques rituelles. 


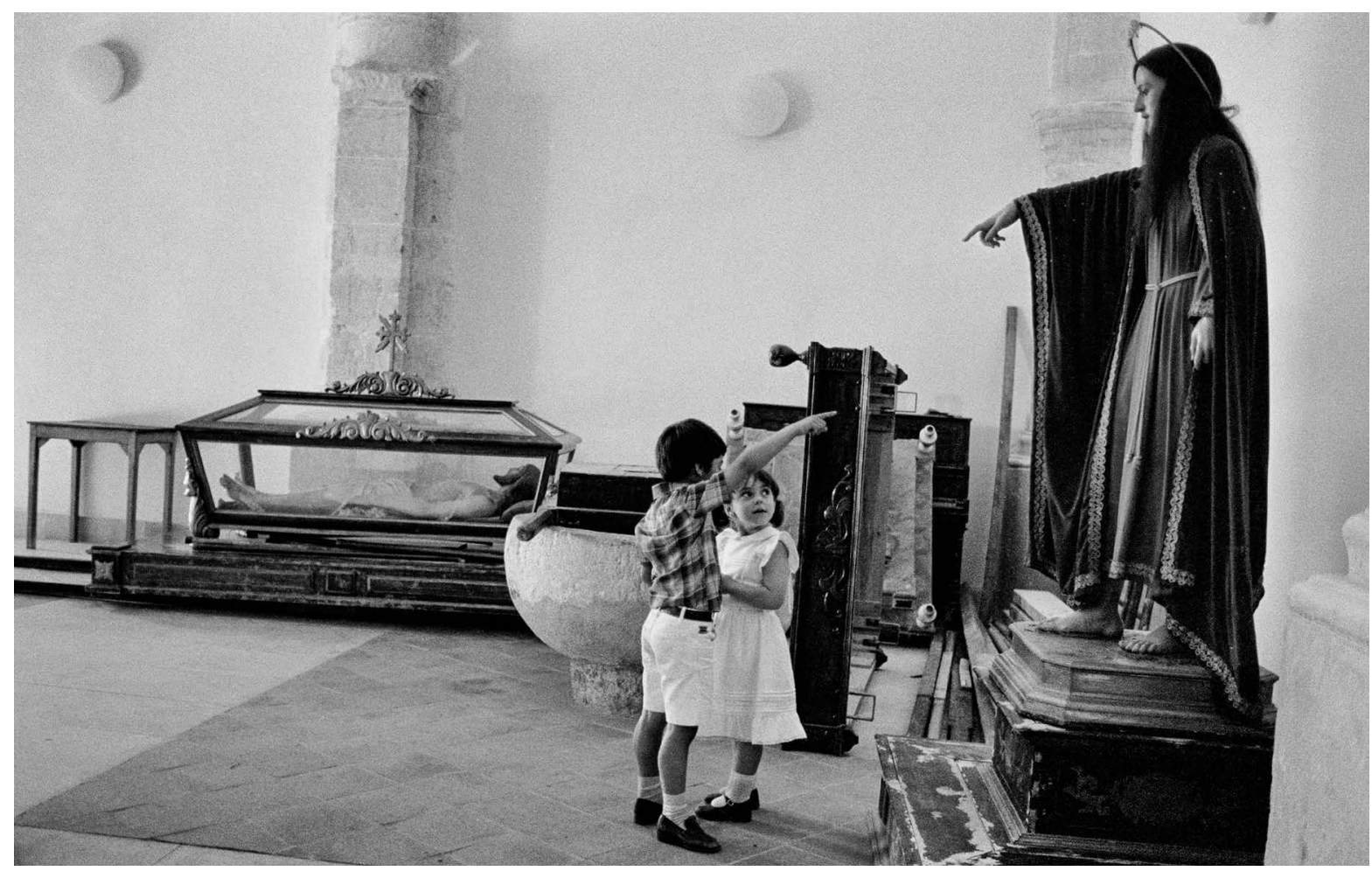

Dans une perspective proche et renouvelée, plusieurs de nos contributions traitent de cette mécanique soma-mnémonique. À différents niveaux, elles montrent comment les sens produisent des savoirs transmissibles, à la manière dont Kenneth $\mathrm{M}$. Kessinger, au sujet des Cashinahua du Pérou, a mis en exergue la "connaissance de la peau» (skin knowledge ou bichi una), la «connaissance du foie» (liver knowledge ou taka una) et encore celle des «oreilles» (ear knowledge ou bapinki una) [cité par Howes 2009: 2]. Ces savoirs renseignent tous sur une réalité sociale externe aux états corporels. Bérénice Gaillemin analyse ainsi comment, en milieu catholique andin, les fidèles apprennent les prières à l'aide de disques d'argile composés de figurines, de fleurs et de matériaux divers tels la laine et le coton, qui transcrivent matériellement le catéchisme. L'apprentissage verbal se convertit par cet objet-texte en un processus synesthésique: il implique, d'abord, le passage du registre sémantique au registre phonétique - chaque figurine transcrit une syllabe - puis la collaboration et la mobilisation de tous les sens. Le pratiquant peut sentir, voir, toucher et goûter le texte. Au Maroc, où Anouk Cohen a effectué son enquête, le Coran est appris aux côtés du professeur à partir de la planche de bois puis du Livre, calligraphiés et enluminés de motifs employés en tant que supports mnémoniques ou aides à l'action rituelle, comme dans le cas des médaillons signalant la prosternation requise à la fin de certains versets. L'apprenti est ici entrainé par un dispositif d'imbrication des sens où l'image du texte se transforme en sonorité religieuse et en posture rituelle. S'intéressant à la communication trans-spécifique des Runa de la forêt amazonienne au Pérou, Andrea-Luz Gutierrez Choquevilca explore l'ambiguïté de la perception acoustique. Elle montre combien la transmission sensorielle des savoirs fig. 2

Cristina Garcia Rodero,

Le doigt de saint Jean,

Espagne, Belinchón, 1982 (C) Cristina Garcia Rodero/ Magnum Photos.

\section{double page}

suivante

\section{fig. 3}

Beniamino Pisati, Baptême orthodoxe à Tbilisi, Géorgie, 2016 (C) Beniamino Pisati. 


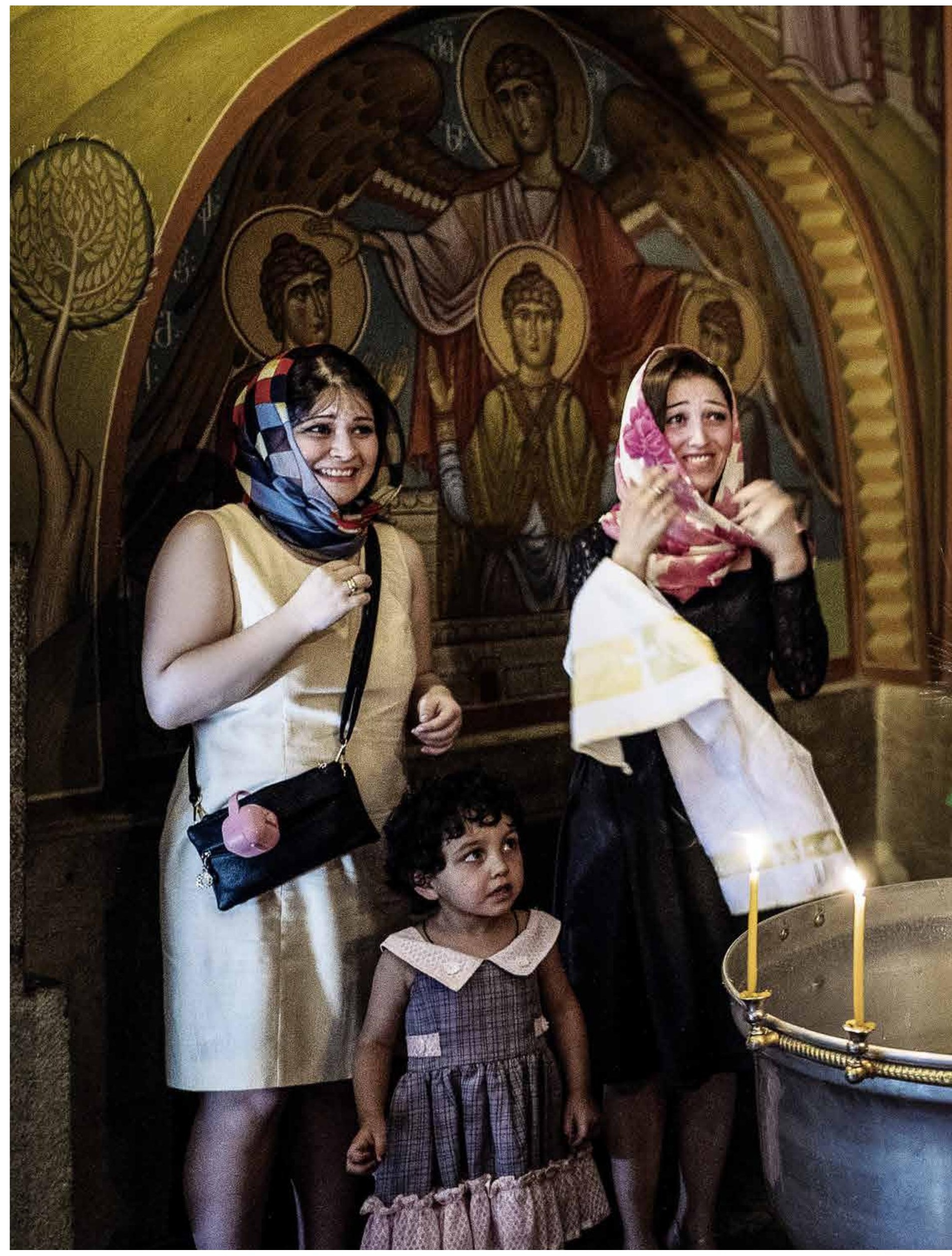




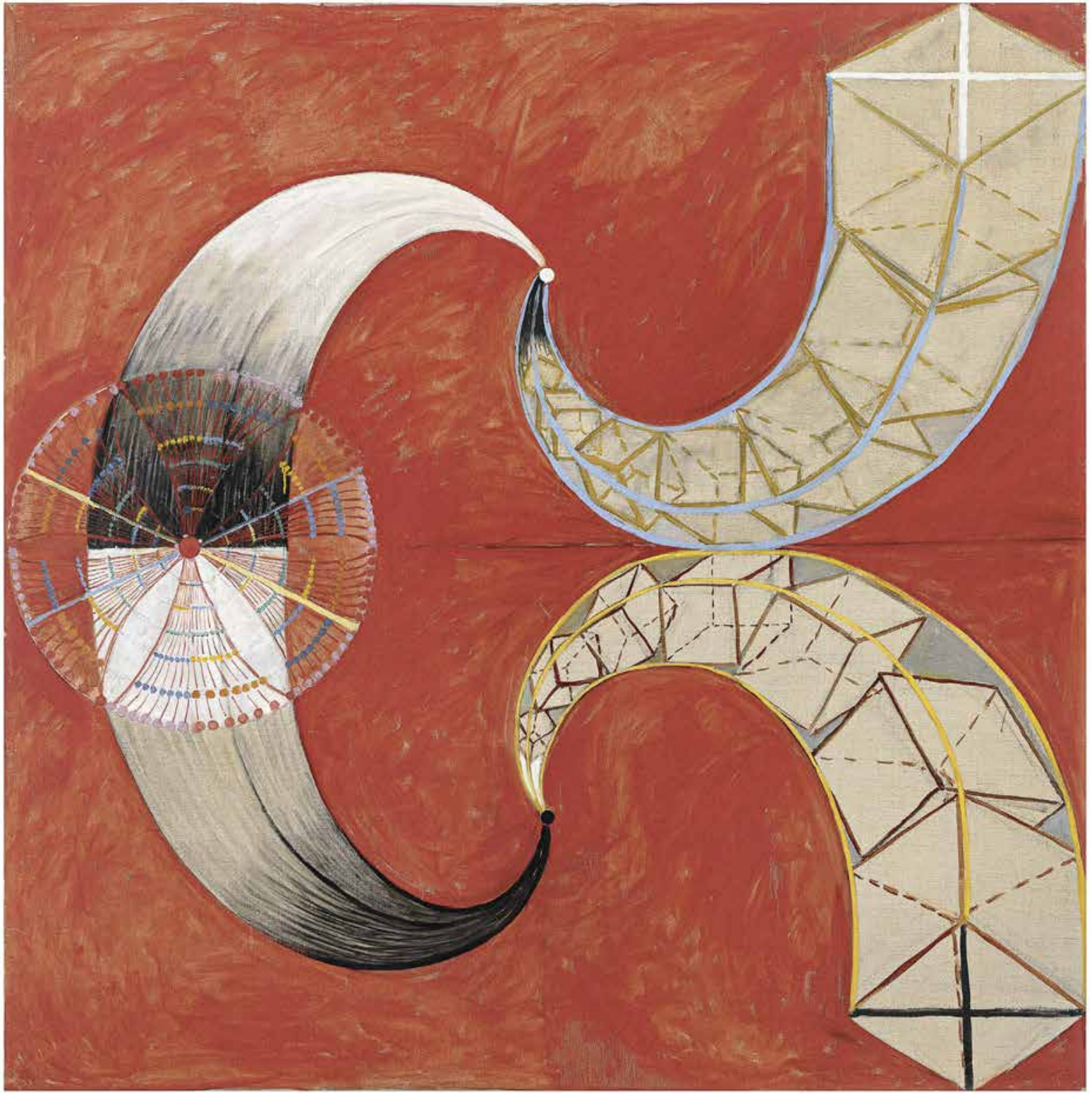

fig. 4

Hilma af Klint,

Grupp IX/SUW, nr. 9,

Svanen nr. 9, 1915.

Avec l'aimable autorisation

de The Hilma af Klint

Foundation (C) Photo: Albin

Dahlström/Moderna

Museet-Stockholm-Suède. 
chamaniques est indissociable d'un imaginaire de la voix qui rend audibles des êtres invisibles et analyse les soubassements cognitifs de cet imaginaire, notamment à travers la mise en récit de la rencontre avec les animaux ou les esprits. Dans le palo monte, Katerina Kerestetzi examine le corps situé comme le lieu d'une cristallisation, sinon d'une concrétisation mémorielle du rituel. Par les gestes localisés dans l'espace du culte, les mouvements déployés à l'échelle du sanctuaire ou encore les regards orientés selon l'agencement architectural de la maison du pratiquant, le corps s'entraîne non seulement à la ritualité, mais s'en imprègne tout comme il en communique les sensations et le sens. Quant à Damien Mottier, son texte sur l'imposition télévisuelle des mains des prédicateurs pentecôtistes rappelle que le corps des spectateurs doit s'ouvrir à la promesse miraculeuse de l'agir divin, autrement dit être réceptif, habitué sinon converti, pour que la performance puisse être éprouvée. L'échec, bien entendu, est parfois de mise et permet de mieux mettre en valeur la mécanique mnémo-somatique de l'expérience.

Fréquemment entendue sur nos différents terrains au sujet de la manifestation tangible de tel dieu ou tel esprit, l'expression «ça me touche" est emblématique de cette mécanique mnémo-somatique. Elle énonce la manière dont les pratiquants éprouvent une présence. Cette sensation physique constitue pour eux une preuve de leur communication personnelle avec les esprits ou le divin et mobilise, le plus souvent, toute une «imagerie sensorielle" (Csordas 2002). II en va ainsi de certains pentecôtistes qui se disent traversés par l'Esprit saint comme par une décharge électrique ou des musulmans qui s'émeuvent de toucher, voir ou entendre le Coran, contenant de la Parole de Dieu. La vue, l'ouïe, l'odorat, le toucher, le goût ou le corps distribué dans l'espace sont, comme le montre ce numéro, activés par toute une série de médiations - un livre, une télévision, un cri, un morceau de laine, un chaudron, etc. - articulées à un environnement physique spécifique (une forêt, une montagne, une église, un marché, une maison) ou socio-technique. L'orchestration matérielle des différentes sensorialités religieuses participe à la transmission du message rituel et modélise la sensibilité et la subjectivité des participants.

\section{Spatialiser: espaces et morphologie}

Les sensations n'émergent pas simplement dans un lieu; bien plus, elles en procèdent. C'est pourquoi l'espace - entendu au sens large de contenant, de surface ou de volume - est au cœur de ce dossier qui adopte le point de vue du pratiquant situé dans un régime collectif de sens. Alessandro Duranti (1992) qualifie l'espace de "carte sociale et culturelle" dans la mesure où il fournit des informations sur la manière d'agir et sur la nature des événements qui s'y déroulent. Pour l'architecte Amos Rapoport, ces indices peuvent générer ou inhiber des actions (1982: 50). Par exemple, dans une église catholique, la présence du crucifix invite et conduit les fidèles à baisser la tête et à faire un signe de croix. Aussi, loin d'être un simple support de symboles, l'espace, appréhendé dans sa matérialité - soit en tenant compte de ses dimensions morphologiques et topologiques -, encadre la perception, impacte les états corporels, prescrit des comportements et conditionne la manière dont les entités spirituelles peuvent être ressenties. Comment les fidèles éprouvent-ils un espace religieux et dans quelle mesure ce qu'ils 
y expérimentent s'ancre-t-il dans une configuration sensorielle partagée? Telle est l'une des questions traitées ici, l'espace religieux étant envisagé comme une réalité partagée et le plus souvent non verbalisée.

Cette dimension mnésique de l'espace permet de dépasser le dualisme entre pensée et sensation et de mieux saisir l'expérience sensible en tant que «cognition distribuée» (distributed cognition) [Hutchins 2001; Gell 1998]. «L'homme pense avec ses doigts », pour reprendre une expression chère à Jean-Pierre Warnier (1999). Phénoménologue de l'espace, le géographe Yi-Fu Tuan écrit: «Voir est penser, dans le sens où il s'agit d'une activité qui distingue et qui construit, en créant des modèles de réalité adaptés à des fins particulières. Même le goût, l'odorat et le toucher sont affectés par la pensée (et la pensée par eux) dans le sens décrit ci-dessus: ils distinguent parmi les stimuli et sont capables d'articuler les mondes gustatifs, olfactifs et tactiles. " (Tuan 1975: 152, trad. des auteurs) La notion d' "espace somatisé » (embodied space) [Low et Lawrence-Zunica 2003] synthétise remarquablement cette combinaison des aspects physiques (le corps biologique des naturalistes, phénoménologues ou cognitivistes) et représentationnels (le corps vécu des culturalistes et des sémioticiens) du corps, voire de l'espace corporel. Cette dernière expression désigne aussi bien les représentations de son propre corps dans un espace donné que l'intériorisation des spatialités externes au corps. Nous souhaitons prolonger ici cette réflexion en montrant comment la sensorialité de l'espace fait partie intégrante de l'expérience des pratiquants et impacte leurs conduites motrices. L'étroitesse ou l'ampleur d'un lieu de culte, la densité de ses odeurs, sa luminosité, son acoustique et sa température, influencent des postures, des expressions faciales et des schèmes interactionnels qui se sédimentent comme des traditions non verbalisées.

En parlant dans ce numéro d'expérience (religieuse) spatiale, nous visons à reconsidérer la notion d'espace. Les surfaces ou volumes «acoustiques», "olfactifs», «auditifs», «thermiques» (ibid.), ou encore les smellscapes et les soundscapes (Porteous 1990; Hirschkind 2006; Rowlands 2007), dilatent ou rétrécissent les frontières d'un espace, en altérant la perception et l'action qui y prennent place. En contexte rituel, les sens parviennent aussi à unifier ou à séparer des espaces et dès lors à créer des formes de spatialité singulières. Un courant frais ou chaud, un son diminué ou amplifié, une couleur claire ou vive, un motif, etc. peuvent tous constituer la preuve d'une présence spirituelle ou d'une réalité parallèle. Comme le note William Hanks (2000; voir aussi Stépanoff 2013), l'espace de la performance rituelle est composé de l'espace immédiat et de l'espace imaginaire, tel que le monde des morts, le royaume de(s) dieu(x) ou encore le cosmos chamanique. Ces auteurs décrivent les indices linguistiques, gestuels et visuels qui permettent aux pratiquants de se représenter leur environnement physique comme un espace partagé entre humains, dieux et esprits. Nous nous inscrivons dans cette réflexion en portant une plus grande attention aux référents sensoriels et à la manière dont chaque environnement rituel les met en scène. La forêt amazonienne - le «village des esprits » selon le terme des Runa étudiés par Andrea-Luz Gutierrez Choquevilca - est ainsi transportée dans l'espace clos des rituels par la simulation phonétique et chantée des cris de certains animaux qui, plus que de les évoquer, fait ressentir leur présence inquiétante. Dans ce lieu décentré en quelque sorte, «muer sa propre voix c'est occuper pragmatiquement une autre place, 


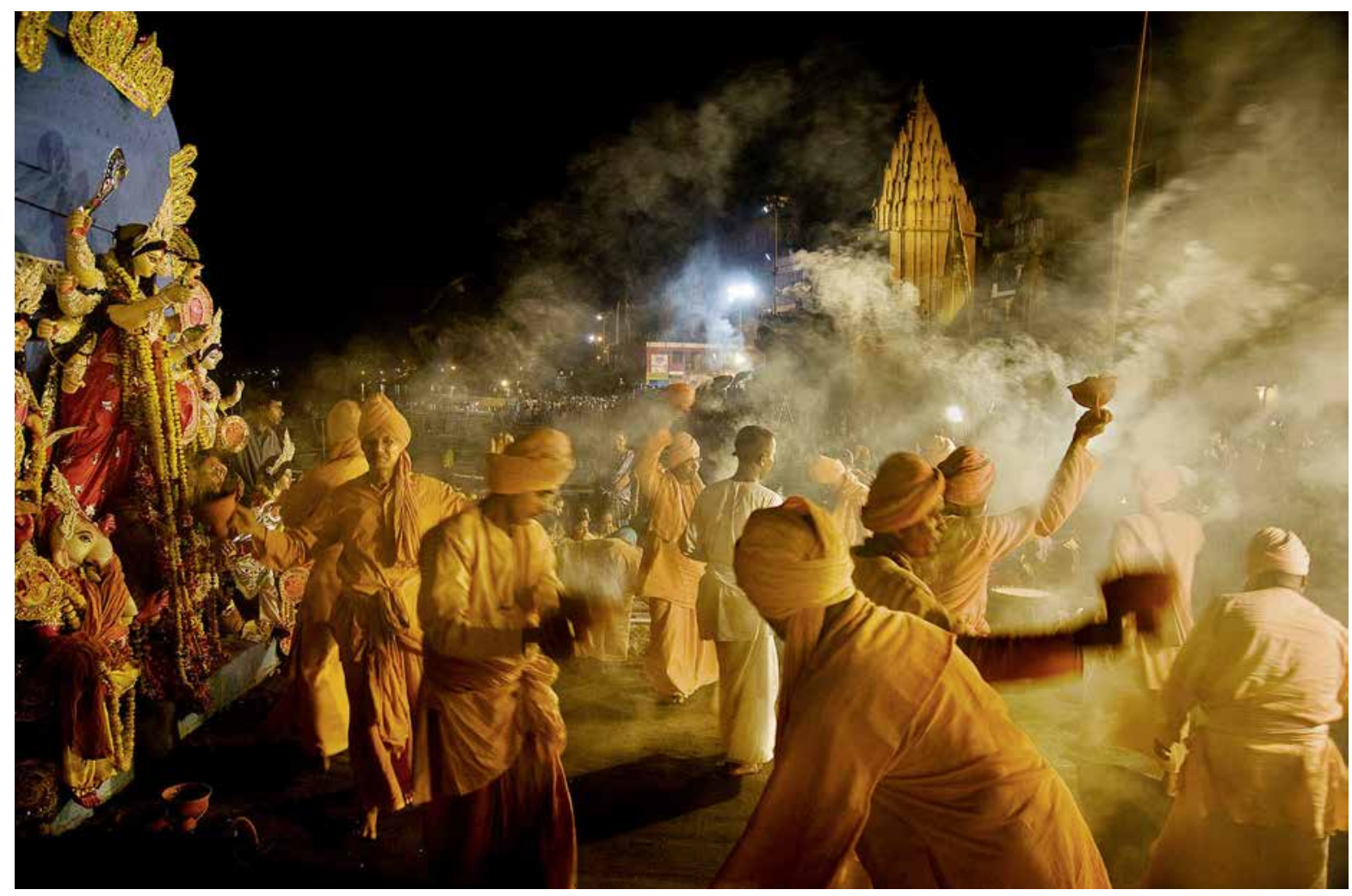

parler d'un autre lieu. » (Gutierrez Choquevilca, ce volume, p.140) Dans les églises catholiques des Andes boliviennes, l'espace formé par les disques d'argile qui transcrivent les prières est un locus où se rencontrent, par un jeu de transmutations perceptives, les lieux mythiques du catholicisme, l'espace immédiat des catéchumènes, le paysage environnant, la Pachamama même: il s'agit, nous dit Bérénice Gaillemain, d'un artefact qui présentifie, de manière sensorielle, l'ensemble de la communauté. Le Coran du souverain marocain réunit iconiquement dans la spatialité de ses pages, lieux sacrés de l'Islam, corporéités royales, postures humbles des fidèles et identité nationale (Cohen, ce volume, p.72). Dans le cas des pentecôtistes étudiés par Damien Mottier, le geste de poser la main sur l'écran d'un téléviseur, afin de recevoir l'effusion de l'Esprit saint promise par un téléprédicateur, réalise un miracle géographique: l'espace domestique du fidèle et l'endroit d'où provient le Saint-Esprit fusionnent.

\section{Régler: sens, discipline et collectif}

Jean-Pierre Warnier a montré comment, au Cameroun, la soumission absolue au roi sacré est intériorisée grâce à l'interaction avec des objets et des substances qui modèlent discrètement la sensibilité des corps appelés à devenir des corps dévotionnels (2009). Ce numéro s'engage sur le même chemin pour penser les liens entre norme, matérialité et sensorialité. La problématique de la normalisation des sens est relativement peu traitée en anthropologie des faits religieux ${ }^{3}$. Pourtant, dans toutes les religions, qu'elles soient ou non institutionnalisées, normaliser et fixer des traditions cultuelles fig. 5

Raghu Rai, Uttar Pradesh,

Bénarès, 2010 @ Raghu

Rai/Magnum Photos.

3. Sur les politiques

du sensoriel en contextes religieux, se reporter notamment à Farag 2009 , Hirschkind 2006 et Murphy 1998. 

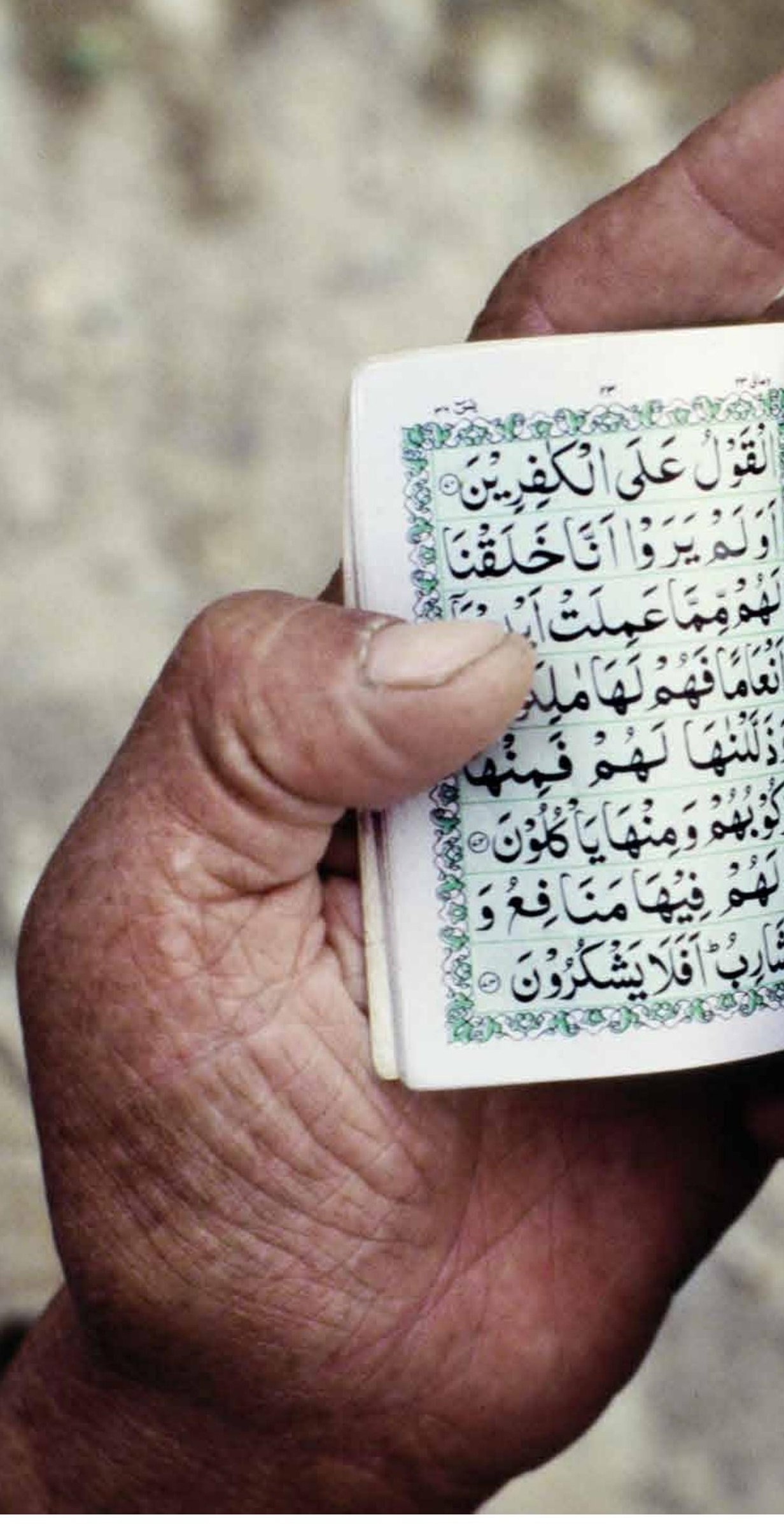
ज-

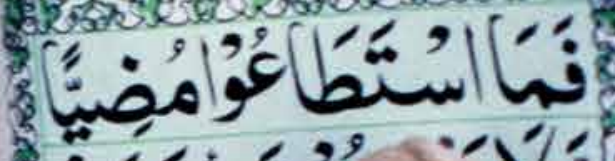

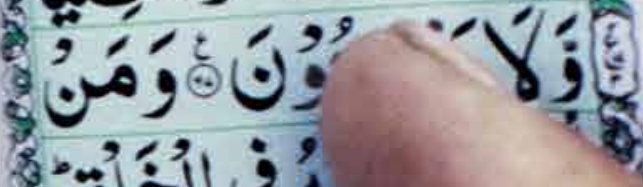

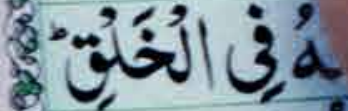

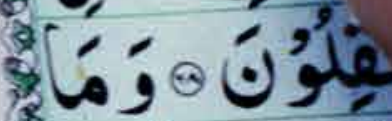

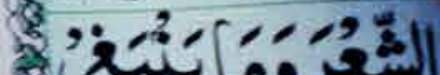

शु. (1)

:

Q

ช)

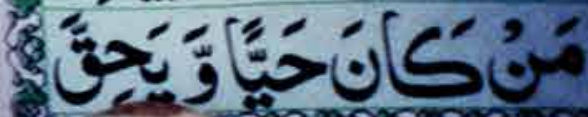

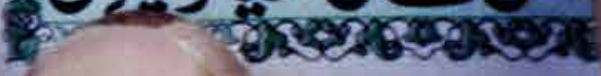

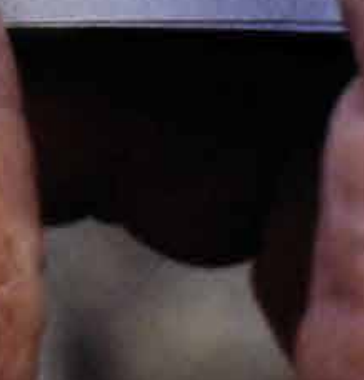


4. La notion d'affordance, propre au courant de la psychologie de la perception, notamment au travail de Gibson (1977), désigne la capacité d'un objet ou d'un espace à suggérer, par sa matérialité, une utilisation qui lui est propre.

\author{
double page \\ précédente \\ fig. 6 \\ Steve McCurry, \\ Afghanistan, 2006 \\ (C) Steve McCurry/Magnum \\ Photos.
}

passe aussi par une orchestration subtile des sens. Le corps y est modelé par un filtrage spécifique des stimuli sensoriels qui peut procéder de démarches involontaires tenant par exemple à l'espace-cadre (frame space) [Goffman 1991] de la pratique religieuse ou bien de mesures politiques.

Au Maroc, explique Anouk Cohen, la normalisation des sensorialités religieuses prend appui sur la reconfiguration matérielle du Coran. Conçu par le monarque comme un dispositif graphique, mnémonique et cognitif, le Livre en tant qu'objet est voué à produire une expérience sensible commune et un mode de connaissance susceptible de transformer concrètement la religiosité des fidèles selon l'orthodoxie et l'orthopraxie officielles pour affirmer l'autorité du roi, «commandeur des croyants». Considérée comme dispositif sensoriel, la religion répond ici à une « technologie politique " (Foucault 1975) des corps et des sens, façonnables selon l'agenda du pouvoir. L'établissement d'une police, au sens de Jacques Rancière, soit «un ordre des corps qui définit les partages entre les modes du faire, les modes d'être et les modes du dire” (1995: 51), cherche à inscrire les pratiquants dans une entité sociale et religieuse reconnue comme leur(re) (Cohen, ce volume, p.72 ). S'agissant des supports circulaires d'argile modelés sur le sol des églises andines - pensés et tracés pour lire et mémoriser la Bible -, leur usage (allant de la confection à leur déchiffrement) est soumis à des règles définies de manière cyclique et rotative par un maestro. Ce dernier est élu par les autorités indigènes locales pour conceptualiser une grammaire visuelle d'images qui donnent forme et corps aux prières, selon des normes inscrites dans une cosmogonie et une écologie propres à chaque communauté (Gaillemin, ce volume, p.46). Dans ce type de contextes, l'établissement différencié de normes sensorielles stabilise, pour un temps, les manières dont les pratiquants entrent en relation avec le divin. Loin d'être privé et confidentiel, ce mécanisme, procédant tant de l'idiosyncrasie religieuse que du partage du sensible, relève donc aussi du sens collectif ou du sens du collectif, ceux-ci pouvant être, tour à tour, normés et normatifs.

La question de la modélisation sensorielle de la praxis et de la subjectivité religieuses doit s'inscrire dans une réflexion plus large sur le rapport du corps à la norme afin de mieux cerner la performativité des sens - par et pour eux-mêmes - dans la configuration dynamique des pratiques rituelles. À partir d'études axées sur des couplages et découplages de régimes sensoriels spécifiques, ce dossier a pour ambition de comprendre comment le système sensoriel, aux prises avec une matière plus ou moins contrôlée, règle le déroulement de l'action. Au cours des rituels, les pratiquants construisent leur rapport sensible autant qu'ils sont définis par lui. Dans le palo monte, par exemple, la taille du sanctuaire, l'ordre ou le désordre qui le caractérisent, l'agencement du décorum, l'effroi suscité par des substances telles que le sang et les restes d'animaux ou encore le dégoût provoqué par les odeurs qui en émanent, ont un impact direct sur les pratiques rituelles. La manière dont celles-ci sont sans cesse façonnées échappe aux pratiquants car c'est leur corps dans un espace qui les font et les défont. Dans ce culte, la norme n'est pas fixée en conscience par les dévots. Elle est principalement établie par la spatialisation de la pratique et l'affordance kinésique ${ }^{4}$ du lieu. L'aménagement personnel de l'espace rituel induit des mouvements, des attitudes et des postures qui, en singularisant l'expérience initiatique et cultuelle de chaque groupe, peuvent se fixer ou dépérir (Kerestetzi, ce volume, p.100). En Bolivie, l'emploi d'objets tangibles pour traduire visuel- 
lement, tactilement et acoustiquement la Bible permet aux pratiquants de mémoriser les doctrines et d'en acquérir la signification. Celle-ci peut être redéfinie par la perception sensorielle sans cesse changeante des «lieuximages" (trans)formés tant par la matérialité instable des disques d'argile qui leur donnent forme que par l'espace où ils prennent place. Les sens, en prise avec la matière, soutiennent donc l'incorporation du texte en même temps qu'ils en modélisent le sens (Gaillemin, ce volume, p.46). Chez les Runa de l'Amazonie péruvienne, la rencontre avec les esprits est marquée par un cadre perceptif singulier: entendre sans voir. Les rêves, les récits, les chants contribuent au déchiffrement de l'intentionnalité de la communication trans-spécifique avec les animaux ou les esprits. Ce travail de traduction est finement analysé par Andrea-Luz Gutierrez Choquevilca à la fois du point de vue de la transformation de la matière phonique et du partage d'une sémiotique basée sur le pouvoir d'action de la voix. Au sujet des usages pentecôtistes de la télévision, Damien Mottier montre que cette technique de médiation est devenue un élément constitutif de l'expérience. La relation aux écrans participe au rituel, modélise les comportements et contribue à la formation des sensibilités individuelles et collectives. À tel point qu'on peut se demander si la transmission télévisuelle des cultes ne constitue pas une forme de liturgie sur laquelle repose en partie l'orthopraxie de cet univers religieux extrêmement fragmenté.

Ainsi, bien plus que récepteur ou performatif (transmetteur de messages), le corps pris dans l'action rituelle compose une «socialité sensorielle" (sensorialized sociality) [Chau 2008: 500] agissante dans la fabrication de l'expérience religieuse, soit un régime collectif de sens.

CNRS/LESC

anouk.cohen@wanadoo.fr

CNRS-LAS

katerina.kerestetzi@college-de-france.fr

Université Paris-Nanterre / Histoire des Arts et des Représentations (HAR) dmottier@parisnanterre.fr 



\section{Stépanoff, Charles}

2013 "Dessins chamaniques et espace virtuel dans le chamanisme khakasse", Gradhiva 17: 144-169.

\section{Stoller, Paul}

1989 The Taste of Ethnographic Things: the Senses in Anthropology. Philadelphie, University

of Pennsylvania Press.

\section{Tuan, Yi-Fu}

1975 «Place: an Experiential Perspective", Geographical Review 65 (2) : 151-165.

\section{Warnier, Jean-Pierre}

1999 Construire la culture matérielle: l'homme qui pensait avec ses doigts. Paris, PUF.

2009 Régner au Cameroun: le roi-pot. Paris, Karthala.

\section{Wathelet, Olivier}

2009 «Anthropologie de la transmission des savoirs et savoir-faire sensoriels. Étude de cas: la transmission d'un patrimoine olfactif à l'intérieur de la famille ". Thèse en anthropologie sociale et ethnologie, université de Nice-Sophia Antipolis.

\section{Whatelet, Olivier et Candau, Joël}

2013 "Considérations

méthodologiques en anthropologie sensorielle: pour une ethnographie cognitive des perceptions (ECP) ", in Joël Candau, Marie-Barbara Le Gonidec (dir.), Paysages sensoriels: essai d'anthropologie de la construction et de la perception de l'environnement sonore. Paris, Éditions du CTHS : 213-239.

\section{Whitehouse, Harvey}

2000 Arguments and lcons: Divergent Modes of Religiosity. Oxford, Oxford University Press.

2004 Modes of Religiosity: a Cognitive Theory of Religious Transmission. Walnut Creek (Canada), AltaMira Press.

page 4 et ci-contre Raghu Rai, Uttar Pradesh Bénarès, 2010 (détail) (c) Raghu Rai/Magnum Photos.

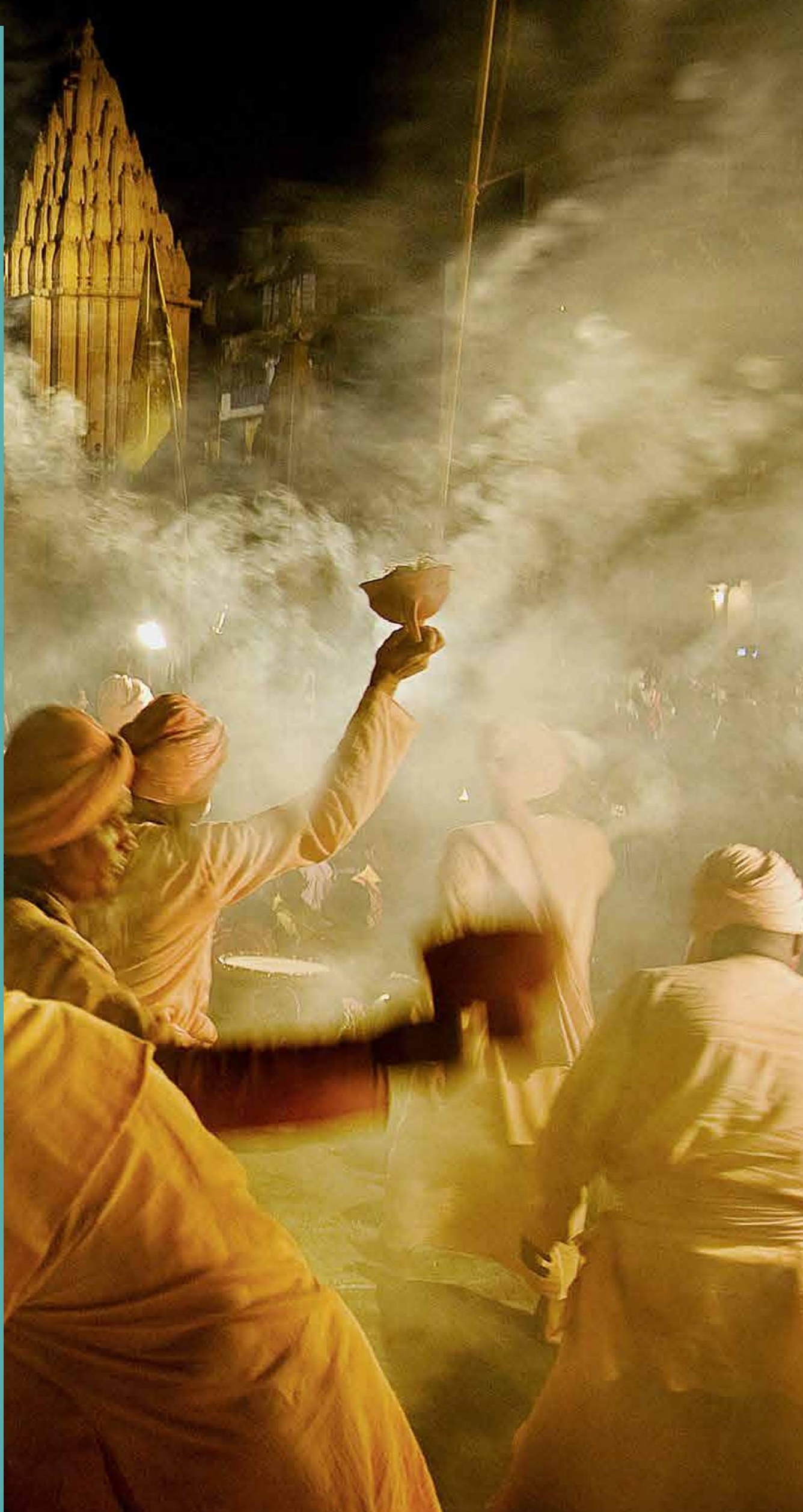

